\title{
PULSATION MODAL BEHAVIOR OF CLASSICAL CEPHEIDS IN THE HR DIAGRAM
}

Arthur N. Cox and Stephen W. Hodson

Los Alamos Scientific Laboratory

Mapping of the Cepheid region of the Hertzsprung-Russell diagram has been difficult to do from a theoretical viewpoint because the masses and compositions of the Cepheids have always been very uncertain. It now appears that the various mass anomalies have been solved by distance scale changes and by the realization of very helium rich convection zones. This helium enrichment is caused by a Cepheid wind which blows away more hydrogen than helium, just as in the solar wind. Now with the evolutionary theory masses, radii, luminosities, and our new composition structures, the predicted blue edges of the instability strip and periods throughout the strip agree very well with observations.

Let me first justify the new unconventional composition structure which is the key to my results. Consider the Cepheids with bumps in their light and velocity curves. Their periods range from 5.5 to perhaps 17 days, but the bumps are most visible between 7 and 10 days. Hertzsprung noticed that the bump was very low on decreasing light at 7 days, was at the peak at about 10 days, and crossed over to rising light at longer periods. Simon and Schmidt (1976) have used Stobie (1969) calculations to show that the ratio of the linear theory second overtone to fundamental period ratio $\Pi_{2} / \Pi_{0}$ is uniquely correlated with bump phase. Thus the phase is late when $\Pi_{2} / \Pi_{0}$ is 0.53 and $\Pi_{0}$ is 5.5 days, and early when $\Pi_{2} / \Pi_{0}$ is 0.46 at 17 days.

The first figure shows the period ratio $\Pi_{2} / \Pi_{0}$ versus period for models with various masses and compositions. First notice the $7 \mathrm{Mo}$ models with the homogeneous King IVa composition. None should give bumps because $\Pi_{2} / \Pi_{0}$ is always above 0.53 . If the $1 u-$ minosity is retained, but the mass decreased to $5 \mathrm{M}_{\odot}$ as Christy suggested, there are predicted bumps for at least the longer periods. Use of the new Carson opacities at $7 \mathrm{M}_{\odot}$ gives bumps at the longer periods as Sastri Vemury (1977) has reported. Actually, we get no bumps at the extension near 9 days using a composite of 
ARTHUR N. COX AND STI:PHI:N W. HODSON

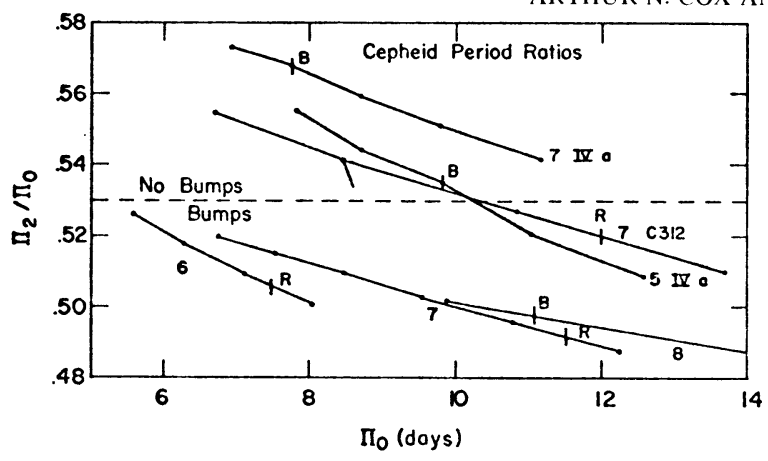

Fig. 1. $\Pi_{2} / \Pi_{0}$ versus $\Pi_{0}$ for Cepheids.

opacities, but at a somewhat larger amplitude, Sastri does get a bump. At 6,7 , and $8 \mathrm{M}$, with $Y=0.75$ in the convection zones redward of the blue (B) and blueward of the red (R) edges, the proper Hertzsprung progression is reproduced.

Next we give our theoretical data. The Sandage and Tammann (1969) blue and red edges are the only observational data here. First. notice the $7 \mathrm{M}_{\beta}$ evolutionary theory track. Next, the masses used are based on an evolutionary theory mass-1uminosity law. Linear theory periods and growth rates have been calculated for many models all over this diagram using the helium enrichment for masses up to $8 \mathrm{M}_{\Theta}$. The wind does not blow long enough for enrichment above this mass where evolution is too rapid. The fundamental, first overtone, and second overtone blue edges are indicated. Nonlinear calculations have the second overtone pulsations at too low an amplitude for visibility in the case of the very blue models.

Comparison of periods with observations is in the last figure. The observational and theoretical blue edges appear again. Agreement is very good, but a few interesting discrepancies still exist. The Cepheid wind helium enrichment in the two mixed convection zones seems to be verified by many observations. More nonlinear calculations are underway.

\section{REFERENCES}

Christy, R.F. (1968). Qtr. J. Roy. Astron. Soc. 2, 13. Sandage, A. and Tammann, G.A. (1969). Astrophys. J. 157, 683. Simon, N.R. and Schmidt, E.G. (1976). Astrophys. J. 205, 162. Stobie, R.S. (1969). Mon. Not. R. Astron. Soc. 144, 485 . Vemury, S.K. (1977). Bull. Am. Astron. Soc. 9, 360 . 


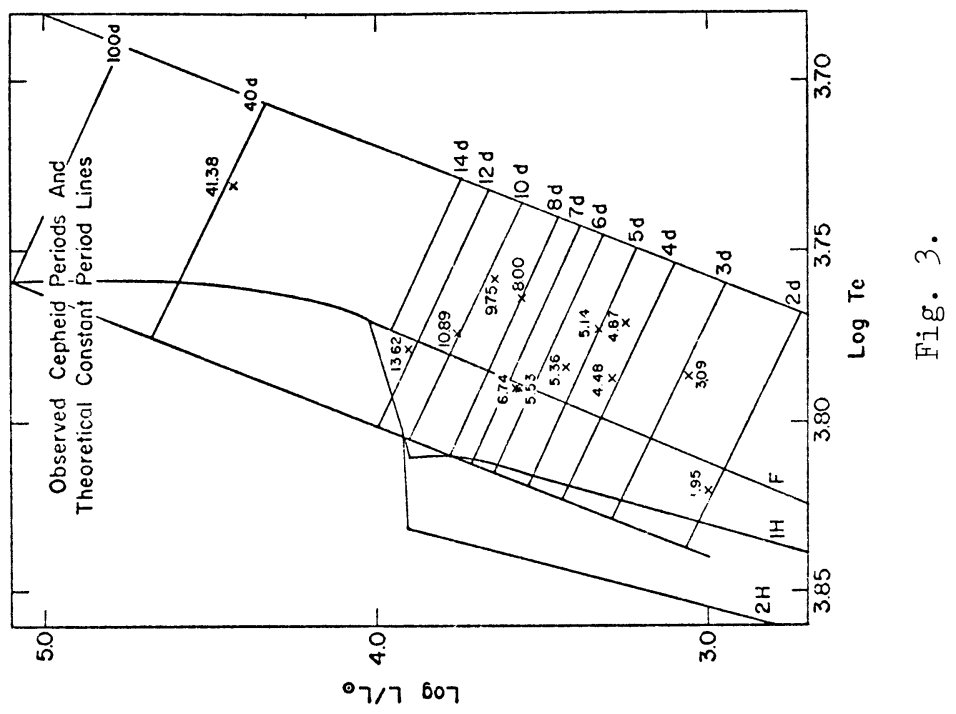

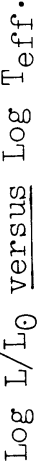




\section{DISCUSSION}

TAYLER: As you know I am worried about whether you could keep a stable high helium content layer on top of a lower helium content interior. There must surely be some instability and mixing. At the least a molecular weight gradient should be set up which is marginally stable. I cannot however make an immediate estimate of the change which this would make in your required $Y$.

COX: You have brought up one of the main problems to this enrichment. This Rayleigh-Taylor instability might be very fast and prevent the enrichment unless the Cepheid wind is very strong. Short time scales may exist but perhaps a gradient of helium can be set up which mixes slowly enough to give helium enrichment and a reconcilation of the Cepheid mass discrepancies. So far I haven't been able to figure out how to do the calculations for this mixing time scale.

MADORE: Instead of the necessity of invoking mixing to explain the presence of Cepheids without bumps it may be that here we are seeing the first-crossing Cepheids where the wind has not yet had time to act. This of course could be checked by comparing the ratio of Cepheids with and without bumps to the ratio of the second and first crossing times.

COX: I think that there may be good agreement here. Perhaps $20 \%$ of the 7-10 day Cepheids do not have bumps, and I believe that the first crossing time is also only about $20 \%$ of the total time that a star is a Cepheid.

WALLERSTEIN: If the helium is increased from about $\mathrm{Y}=0.25$ to 0.75 , X must have decreased from about 0.72 to 0.22 . The effect of this is to increase the metals/hydrogen ratio by a factor of 3 to 4. So far as I know, no such effect has been seen in the comparison of Cepheids with non-variable supergiants.

COX: Of course I don't know if the effect of large changes of $\mathrm{Z} / \mathrm{X}$ is observable. Remember that all second crossing yellow giants hotter than about $4500 \mathrm{~K}$ would have the same effect regardless of whether they pulsate. Construction of a few good model atmospheres could probably answer the question, but, so far, the only ones I know of are by Parsons without properly allowing for blanketing. I belive factors of two or more in $\mathrm{Z} / \mathrm{X}$ have been easily possible.

APPENZELLER: Assuming your rather high mean molecular weight in the outermost layers of the Cepheids one obviously should expect some change of the stellar radii (as compared to "conventional" models). How big is this effect quantitatively?

COX: In our models we select $\mathrm{L}$ and $\mathrm{T}$ eff and therefore we hold $\mathrm{R}$ fixed for different structures with different helium enrichments. The changes in the periods are due to the changes in the pulsation constant $Q_{i}=\Pi_{i}\langle p\rangle /\left\langle p_{\odot}\right\rangle$ which came about due to different mass concentrations. Periods do change roughly 5-10\% making very large relative changes in $\pi_{2} / \Pi_{0}$ and $\pi_{1} / \Pi_{0}$. 